\title{
A Successful Treatment of Encapsulating Peritoneal Sclerosis in an Adolescent Boy on Long-term Peritoneal Dialysis: A Case Report
}

\author{
Zlatan Zvizdic ${ }^{1}$, Angela Summers ${ }^{2}$, Zia Moinuddin ${ }^{2}$, David Van Dellen ${ }^{2}$, \\ Irmina Pasic-Sefic ${ }^{3}$, Faruk Skenderi ${ }^{4}$, Semir Vranic ${ }^{5}$, Titus Augustine ${ }^{2}$ \\ ${ }^{1}$ Department of Pediatric Surgery, University Clinical Center Sarajevo, Sarajevo, \\ Bosnia and Herzegovina; \\ ${ }^{2}$ Department of Transplant and Endocrine Surgery, Manchester Royal Infirmary, \\ Manchester University Foundation Trust; An United Kingdom National Specialized \\ Centre for Surgery for Encapsulating Peritoneal Sclerosis, Manchester, United Kingdom; \\ ${ }^{3}$ Department of Radiology, University Clinical Center Sarajevo, Sarajevo, Bosnia \\ and Herzegovina; \\ ${ }^{4}$ Department of Pathology, University Clinical Center Sarajevo, Sarajevo, Bosnia \\ and Herzegovina; \\ ${ }^{5}$ College of Medicine, QU Health, Qatar University, Qatar
}

Received April 10, 2020; Accepted November 2, 2020.

Key words: Encapsulating sclerosing peritonitis - Peritoneal dialysis - Management

Abstract: Encapsulating peritoneal sclerosis (EPS) is a rare life-threatening complication associated with peritoneal dialysis (PD). EPS is characterized by progressive fibrosis and sclerosis of the peritoneum, with the formation of a membrane and tethering of loops of the small intestine resulting in intestinal obstruction. It is very rare in children. We present a case of a 16-year-old adolescent boy who developed EPS seven years after being placed on continuous ambulatory peritoneal dialysis (CAPD) complicated by several episodes of bacterial peritonitis. The diagnosis was based on clinical, radiological, intraoperative and histopathological findings. The patient was successfully treated with surgical enterolysis. During a 7-year follow-up, there have been no further episodes of small bowel obstruction documented. He still continues to be on regular hemodialysis and is awaiting a deceased donor kidney transplant. EPS is a long-term complication of peritoneal dialysis and is typically seen in adults. Rare cases may be seen in the pediatric population and require an appropriate surgical approach that is effective and lifesaving for these patients.

Mailing Address: Semir Vranic, MD., PhD., College of Medicine, QU Health, Qatar University, Qatar; e-mail: semir.vranic@gmail.com 


\section{Introduction}

First described in 1980 (Gandhi et al., 1980), encapsulating peritoneal sclerosis (EPS) is a rare chronic inflammatory condition characterized by the presence of fibrosis and adhesions of the peritoneum to loops of the small intestine resulting in intermittent, acute or sub-acute gastrointestinal obstruction (Augustine et al., 2009). EPS has been primarily described secondary to treatment with peritoneal dialysis (PD) but it may also occur as a manifestation of several other conditions, such as systemic autoimmune diseases, diseases of the gastrointestinal tract, peritoneal and intra-abdominal malignancies, exposure to talc or particulate matter or the use of intraperitoneal disinfectant for peritoneal lavage and $\beta$-blocker administration (Plum et al., 2001; Kawanishi and Moriishi, 2005). EPS has also been reported after organ transplantation suggesting that transplantation may be an additional trigger for its onset (Fieren et al., 2007). Etiological factors that cause EPS in cases of long-term PD are insufficiently clear but there is evidence that high glucose concentration and acidic $\mathrm{pH}$ in dialysis fluid, as well as the heat sterilization of PD fluids, cause peritoneal damage and formation of deposits comprising glucose degradation products (GDP) and advanced glycation end products (AGEs) (Jorres et al., 1992; Fieren et al., 2007; Schmitt et al., 2007; Jagirdar et al., 2019). This may be aggravated by recurrent attacks of peritonitis. The reported prevalence of EPS in patients undergoing PD varies from $0.7 \%$ and $3.3 \%$ and increases with length of time on PD (Schmitt et al., 2007; Brown et al., 2009). Clinical presentation of EPS includes weight loss, nausea, anorexia, intractable anemia, hypoalbuminemia, raised inflammatory markers and recurrent episodes of acute or sub-acute small-bowel obstruction. Occasionally, an abdominal mass, formed of the cocooned gut is palpable (Kawaguchi et al., 2000; Brown et al., 2009). The current management of EPS is based on prevention and treatment of inflammatory and fibrotic changes on the peritoneal membrane by immunosuppressive and antifibrotic agents, respectively (Kawaguchi et al., 2000). However, the optimal treatment for EPS remains controversial. Surgery forms an important aspect in the management of EPS and includes excision of the thickened and restricting membrane with enterolysis. Occasionally small bowel resection with the formation of stomas may be required. Postoperative medical treatment, in the form of steroids, tamoxifen or immunosuppression is also used (Celicout et al., 1998; Samarasam et al., 2005; Liu et al., 2009; Cornelis and Oreopoulos, 2011). Regardless of the treatment modality, the mortality rate for patients with EPS is still high and ranges between 25 and 55\% (Kawanishi and Moriishi, 2005; Brown et al., 2009).

Herein, we report a case of a 16-year-old adolescent boy who developed EPS seven years after being placed on PD complicated by several episodes of bacterial peritonitis and who was successfully treated with surgical enterolysis.

\section{Case report}

We report a case of a 16-year-old adolescent boy with a history of chronic renal failure due to bilateral high-grade vesicoureteral reflux and neuropathic bladder who 

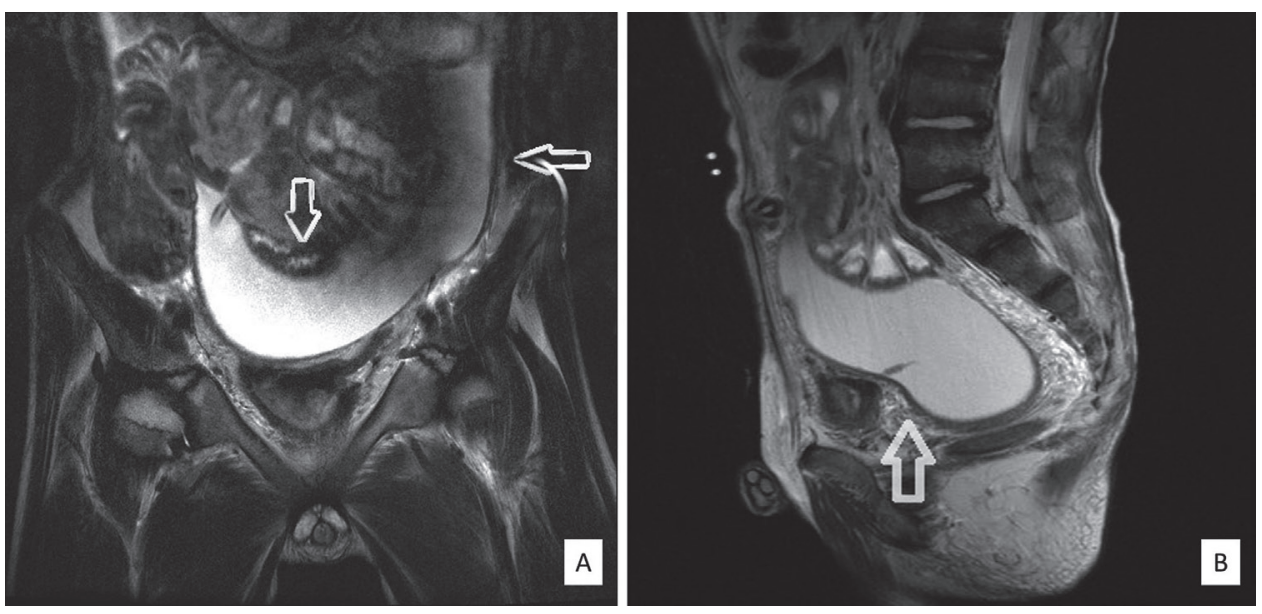

Figure $1 A$ and $B$ - Magnetic resonance imaging T2 WI scans in coronal and sagittal positions demonstrated thickened enhancing peritoneum and hyperintense, loculated fluid collection in the loops of the small bowel (white arrows).

underwent diversionary surgery followed by subtotal bladder excision and bladder augmentation cystoplasty with the sigmoid at the age of 7 years. Two years later, peritoneal dialysis was commenced for renal replacement and associated with several episodes of bacterial peritonitis. After seven years, due to ultrafiltration failure, the PD catheter was taken out and hemodialysis (HD) commenced. Three months after the removal of the peritoneal catheter the patient developed abdominal symptoms consisting of distension and diffuse abdominal pain, associated with loss of appetite, nausea, and intermittent vomiting. It was noted that his weight had reduced by $10 \mathrm{~kg}$ the past year.

A plain X-ray of the abdomen showed multiple dilated loops of small bowel with air-fluid levels indicating an obstruction. An abdominal ultrasound demonstrated an echogenic thickening of the bowel walls of uncertain etiology while MRI (magnetic resonance imaging) indicated a thickened peritoneum and hyperintense, loculated fluid collections between the loops of the small bowel (Figure 1A and B).

After a short course of the conservative treatment that consisted of close monitoring, nasogastric suction, and intravenous hydration, the patient underwent a laparotomy that was performed through the anterior approach with a long midline incision. A thorough abdominal exploration was carried out. The small bowel was covered with a thick cocoon that could not be separated from the intestinal wall. During the procedure there was a perforation of the mid ileum which was repaired with seromuscular single-layered sutures. The findings were consistent with EPS (Figure 2A).

Biopsy of the thickened peritoneum was done during laparotomy and the specimen was submitted to histopathology. It confirmed a thick fibrous capsule that 
was composed of scattered fibroblasts, fibrinous material with dense inflammatory infiltrates of both mononuclear and polymorphonuclear cells.

During the post-operative course there was development of an enteric fistula. Total parenteral nutrition was established and the patient was transferred to the specialized tertiary center for further treatment.

After a two-week period of stabilization with total parenteral nutrition, regular dialysis and antibiotics, the patient was re-explored. Through a long midline laparotomy, the abdominal cavity was carefully entered. The entire small gut was encased in a granulating and fibrotic visceral peritoneal cocoon. There was a cavity anterior to the cocoon extending into the left hypochondrium, containing feculent material. A punched out everted perforation was seen in the cocooned mass. With careful dissection, the encasing cocoon was dissected off the bowel wall, releasing
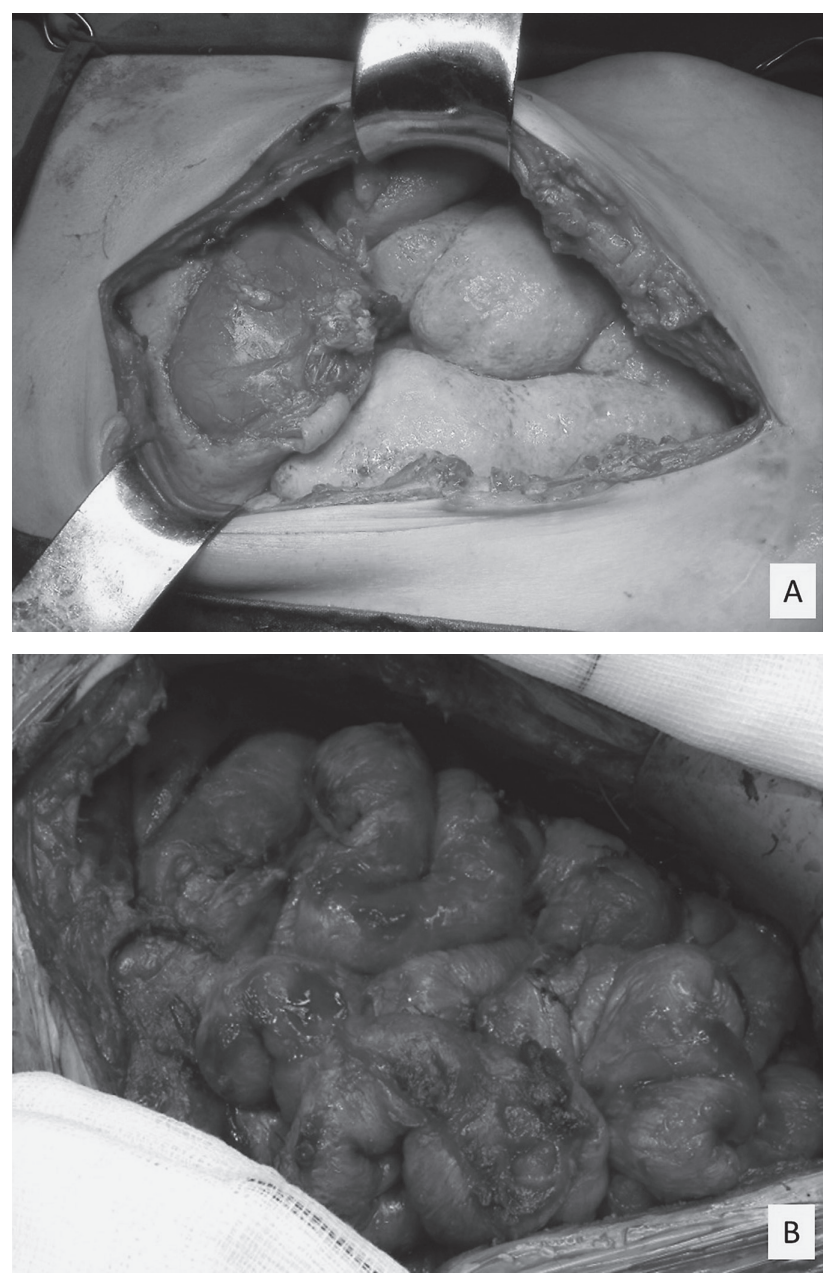

Figure 2 - Gross appearance of encapsulating peritoneal sclerosis before and after enterolysis: peritoneal thickening on the visceral peritoneum of the loops of the small bowel (A) and complete enterolysis (B). 
loops of cocooned small bowel. The entire small bowel was released from the ligament of Treitz to the ileocecal junction. The perforation was localized to the mid ileum. An iatrogenic enterotomy was made in the terminal ileum, which was closed in a single layer. Once a complete enterolysis had been carried out (Figure 2B), the perforation was excised and was brought out in the left iliac fossa as a double-barreled ileostomy. The abdomen was thoroughly lavaged with saline and the abdomen packed with betadine packs. The patient was kept ventilated in the pediatric ICU (intensive care unit) and after 48 hours, re-explored. The gut was found to be healthy without any obvious perforations. The abdomen was closed with interpositioned biologic mesh, Strattice ${ }^{T M}$ with skin closure over the mesh after undermining subcutaneous tissue. A vacuum dressing was placed over the closure. The patient was then woken up gradually and waned off the ventilator after a further 48 hours. There was a 6-week period of recuperation, with wound sepsis with Vancomycin-Resistant Enterococcus (VRE) and Candida, which required micafungin for 3 weeks. A full oral diet was established after 10 days and parenteral nutrition weaned off.

After 6 months, the patient returned to the specialized tertiary center for planned elective closure of the stoma. A midline laparotomy was carried out and the abdomen inspected. The gut was found to be healthy without any evidence of cocooning or adhesions. The double barrel stoma was taken down and closed in a single layer after excising the mucocutaneous edges. The abdomen was closed.

After initially doing well, the patient developed an enterocutaneous fistula by the end of a week, draining about $500 \mathrm{ml}$ of enteric contents towards the bottom of the laparotomy incision. This was treated conservatively by parenteral nutrition for about 8 weeks at the end of which the fistula completely settled and he was established on a full oral diet. He rapidly put on weight after that, with normal nutritional and inflammatory markers.

During a 7-year follow-up, there have been no further episodes of small bowel obstruction documented. He still continues to be on regular hemodialysis and is awaiting a deceased donor kidney transplant.

\section{Discussion}

EPS has been primarily described secondary to treatment with peritoneal dialysis (PD) in adults (Plum et al., 2001; Kawanishi and Moriishi, 2005). It is very uncommon in pediatric population and there are only very few reported and well documented cases of EPS in children (Hoshii et al., 2000; Ekim et al., 2005; Tan et al., 2005). However, the reported outcome of pediatric EPS cases is significantly better with a lower mortality and morbidity compared with adult patients (Shroff et al., 2013; Jagirdar et al., 2019).

Regardless the patient's age and the underlying factors that cause EPS, the principles of management remain the same. Our case illustrates the morbidity that could be associated with this difficult condition and the importance of early definitive diagnosis and effective multidisciplinary surgical intervention. 
For the proper treatment of EPS, Nakamoto (2005) proposed that the development of EPS should be divided based on abdominal symptoms, inflammation, encapsulation, and intestinal findings into four stages: stage 1 (pre-EPS), stage 2 (inflammatory), stage 3 (encapsulating), and stage 4 (chronic EPS).

There is a strong evidence that the duration of the PD therapy is linked with the development of EPS (Kawanishi et al., 2004; Brown et al., 2009). While the length of time alone may not be the strongest factor, that in combination with recurrent episodes of peritonitis and other predisposing factors in all likelihood causes overt disease. It has also been noted that more than two-thirds of EPS cases are diagnosed after discontinuation of PD therapy (Kawanishi et al., 2004), or a modality shift; i.e. PD to HD or PD to successful transplantation. Yamamoto et al. (2010) have suggested that retaining the PD catheter and regular lavage may be a possible limiting factor for the accumulation of factors that encourage the development of the disease. Our case reflects all these etiologic aspects because the presentation of EPS occurred seven years after the introduction of PD therapy and three months after discontinuation of PD.

No gold standard exists for the diagnosis of EPS. At present, there are no reliable predictive markers for EPS. However, in any patient on long term PD with abdominal symptoms, persistently raised inflammatory markers and a declining albumin, the diagnosis of EPS should be considered. The diagnosis of EPS is based on a constellation of clinical findings and confirmed by imaging techniques (CT - computed tomography, or ultrasound) or by laparotomy (Nakamoto, 2005; Moinuddin et al., 2014). Consequently, there are no consensus guidelines on the management of EPS. The mainstay of management consists of the termination of PD and the use of immunosuppressive, antifibrotic medicines, aggressive nutritional support, and surgical treatment when necessary. There is no definite medical therapy for the disease although some small case studies showed the usefulness of corticosteroids, particularly in the treatment of EPS in its early inflammatory stages, and tamoxifen (Korte et al., 2011; Kawanishi, 2012). However, other studies did not confirm the significant utility of tamoxifen, a selective estrogen receptor modulator with strong anti-fibrotic properties related to inhibition of cytokine TGF- $\beta$ (transforming growth factor- $\beta$ ) (Balasubramaniam et al., 2009). Surgical treatment in EPS was previously associated with high morbidity and mortality rates (Kittur et al., 1990). However, more recently, surgical enterolysis has been shown to be the definitive therapeutic option in almost all patients with advanced EPS with acute or subacute intestinal obstruction (Kawanishi et al., 2005, 2011). One of the more important reasons for this increased success is the conduct of treatment by experienced surgeons familiar with this pathology. This therapeutic approach appeared to work in our pediatric case, as the patient had no more recurrent episodes of intestinal obstruction. The management of our patient reflects the challenges that can be faced in the surgical treatment of EPS. It also shows that EPS may affect children and that an aggressive surgical management can be effective 
and lifesaving for these patients. Additionally, this report could alert pediatric nephrologists to this rare but a life-threatening complication of chronic PD and emphasize the need for prompt diagnosis and timely conversion to hemodialysis (HD) in high-risk patients with ultrafiltration failure.

\section{References}

Augustine, T., Brown, P. W., Davies, S. D., Summers, A. M., Wilkie, M. E. (2009) Encapsulating peritoneal sclerosis: clinical significance and implications. Nephron Clin. Pract. 111(2), c149-154; discussion c154.

Balasubramaniam, G., Brown, E. A., Davenport, A., Cairns, H., Cooper, B., Fan, S. L., Farrington, K., Gallagher, H., Harnett, P., Krausze, S., Steddon, S. (2009) The Pan-Thames EPS study: Treatment and outcomes of encapsulating peritoneal sclerosis. Nephrol. Dial. Transplant. 24(10), 3209-3215.

Brown, M. C., Simpson, K., Kerssens, J. J., Mactier, R. A.; Scottish Renal Registry (2009) Encapsulating peritoneal sclerosis in the new millennium: a national cohort study. Clin. J. Am. Soc. Nephrol. 4(7), $1222-1229$.

Celicout, B., Levard, H., Hay, J., Msika, S., Fingerhut, A., Pelissier, E. (1998) Sclerosing encapsulating peritonitis: Early and late results of surgical management in 32 cases. French Associations for Surgical Research. Dig. Surg. 15(6), 697-702.

Cornelis, T., Oreopoulos, D. G. (2011) Update on potential medical treatments for encapsulating peritoneal sclerosis; human and experimental data. Int. Urol. Nephrol. 43(1), 147-156.

Ekim, M., Fitoz, S., Yagmurlu, A., Ensari, A., Yuksel, S., Acar, B., Ozcakar, Z. B., Kendirli, T., Bingoler, B., Yalcinkaya, F. (2005) Encapsulating peritoneal sclerosis in paediatric peritoneal dialysis patients. Nephrology (Carlton) 10(4), 341-343.

Fieren, M. W., Betjes, M. G., Korte, M. R., Boer, W. H. (2007) Posttransplant encapsulating peritoneal sclerosis: a worrying new trend? Perit. Dial. Int. 27(6), 619-624.

Gandhi, V. C., Humayun, H. M., Ing, T. S., Daugirdas, J. T., Jablokow, V. R., Iwatsuki, S., Geis, W. P., Hano, J. E. (1980) Sclerotic thickening of the peritoneal membrane in maintenance peritoneal dialysis patients. Arch. Intern. Med. 140(9), 1201-1203.

Hoshii, S., Honda, M., Itami, N., Oh, S., Matsumura, C., Moriya, S., Mori, M., Hatae, K., Ito, Y., Karashima, S. (2000) Sclerosing encapsulating peritonitis in pediatric peritoneal dialysis patients. Pediatr. Nephrol. 14(4), 275-279.

Jagirdar, R. M., Bozikas, A., Zarogiannis, S. G., Bartosova, M., Schmitt, C. P., Liakopoulos, V. (2019)

Encapsulating peritoneal sclerosis: Pathophysiology and current treatment options. Int. J. Mol. Sci. 20(22), 5765.

Jorres, A., Topley, N., Gahl, G. M. (1992) Biocompatibility of peritoneal dialysis fluids. Int. J. Artif. Organs 15(2), 79-83.

Kawaguchi, Y., Kawanishi, H., Mujais, S., Topley, N., Oreopoulos, D. G. (2000) Encapsulating peritoneal sclerosis: Definition, etiology, diagnosis, and treatment. International Society for Peritoneal Dialysis Ad Hoc Committee on Ultrafiltration Management in Peritoneal Dialysis. Perit. Dial. Int. 20, S43-S55 (Suppl. 4).

Kawanishi, H. (2012) Surgical and medical treatments of encapsulation peritoneal sclerosis. Contrib. Nephrol. 177, 38-47.

Kawanishi, H., Moriishi, M. (2005) Epidemiology of encapsulating peritoneal sclerosis in Japan. Perit. Dial. Int. 25, S14-S18 (Suppl. 4).

Kawanishi, H., Kawaguchi, Y., Fukui, H., Hara, S., Imada, A., Kubo, H., Kin, M., Nakamoto, M., Ohira, S., Shoji, T. (2004) Encapsulating peritoneal sclerosis in Japan: a prospective, controlled, multicenter study. Am. J. Kidney Dis. 44(4), 729-737. 
Kawanishi, H., Watanabe, H., Moriishi, M., Tsuchiya, S. (2005) Successful surgical management of encapsulating peritoneal sclerosis. Perit. Dial. Int. 25, S39-S47 (Suppl. 4).

Kawanishi, H., Shintaku, S., Moriishi, M., Dohi, K., Tsuchiya, S. (2011) Seventeen years' experience of surgical options for encapsulating peritoneal sclerosis. Adv. Perit. Dial. 27, 53-58.

Kittur, D. S., Korpe, S. W., Raytch, R. E., Smith, G. W. (1990) Surgical aspects of sclerosing encapsulating peritonitis. Arch. Surg. 125(12), 1626-1628.

Korte, M. R., Fieren, M. W., Sampimon, D. E., Lingsma, H. F., Weimar, W., Betjes, M. G.; investigators of the Dutch Multicentre EPS Study (2011) Tamoxifen is associated with lower mortality of encapsulating peritoneal sclerosis: results of the Dutch Multicentre EPS Study. Nephrol. Dial. Transplant. 26(2), 691-697.

Liu, H. Y., Wang, Y. S., Yang, W. G., Yin, S. L., Pei, H., Sun, T. W., Wang, L. (2009) Diagnosis and surgical management of abdominal cocoon: results from 12 cases. Acta Gastroenterol. Belg. 72(4), 447-449.

Moinuddin, Z., Summers, A., Van Dellen, D., Augustine, T., Herrick, S. E. (2014) Encapsulating peritoneal sclerosis - A rare but devastating peritoneal disease. Front. Physiol. 5, 470.

Nakamoto, H. (2005) Encapsulating peritoneal sclerosis - A clinician's approach to diagnosis and medical treatment. Perit. Dial. Int. 25, S30-S38 (Suppl. 4).

Plum, J., Hermann, S., Fusshöller, A., Schoenicke, G., Donner, A., Röhrborn, A., Grabensee, B. (2001) Peritoneal sclerosis in peritoneal dialysis patients related to dialysis settings and peritoneal transport properties. Kidney Int. Suppl. 78, S42-S47.

Samarasam, I., Mathew, G., Sitaram, V., Perakath, B., Rao, A., Nair, A. (2005) The abdominal cocoon and an effective technique of surgical management. Trop. Gastroenterol. 26(1), 51-53.

Schmitt, C. P., von Heyl, D., Rieger, S., Arbeiter, K., Bonzel, K. E., Fischbach, M., Misselwitz, J., Pieper, A. K., Schaefer, F.; Mid European Pediatric Peritoneal Dialysis Study Group (MEPPS) (2007) Reduced systemic advanced glycation end products in children receiving peritoneal dialysis with low glucose degradation product content. Nephrol. Dial. Transplant. 22(7), 2038-2044.

Shroff, R., Stefanidis, C. J., Askiti, V., Edefonti, A., Testa, S., Ekim, M., Kavaz, A., Ariceta, G., Bakkaloglu, S., Fischbach, M., Klaus, G., Zurowska, A., Holtta, T., Jankauskiene, A., Vondrak, K., Vande Walle, J., Schmitt, C. P., Watson, A. R.; European Paediatric Dialysis Working Group (2013) Encapsulating peritoneal sclerosis in children on chronic PD: A survey from the European Paediatric Dialysis Working Group. Nephrol. Dial. Transplant. 28(7), 1908-1914.

Tan, F. L., Loh, D., Prabhakaran, K. (2005) Sclerosing encapsulating peritonitis in a child secondary to peritoneal dialysis. J. Pediatr. Surg. 40(5), e21-e23.

Yamamoto, T., Nagasue, K., Okuno, S., Yamakawa, T. (2010) The role of peritoneal lavage and the prognostic significance of mesothelial cell area in preventing encapsulating peritoneal sclerosis. Perit. Dial. Int. 30(3), 343-352. 